Mateusz Pajdziński, Paulina Młynarczyk, Joanna Miłkowska-Dymanowska, Adam J. Białas, Moin Aldin Muhammad Afzal, Wojciech J. Piotrowski, Paweł Górski

Department of Pneumology and Allergy, $1^{\text {st }}$ Chair of Internal Medicine, Medical University of Lodz, Łódź, Poland

\title{
Kyphoscoliosis — what can we do for respiration besides NIV?
}

The authors declare no financial disclosure

\begin{abstract}
Kyphoscoliosis (KS) is a significant clinical problem with no precise guidelines for management, especially concerning respiratory pathology. No exhaustive systematic review has yet been performed. The aim was to conduct a systematic review of available data concerning the pathophysiology and treatment of kyphoscoliosis.

An electronic systematic search compliant with Preferred Reporting Items for Systematic Reviews and Meta-Analyses (PRISMA) guidelines was conducted. The Pubmed database was examined and the search was updated to November 10, 2016. In total, 188 articles were screened, and 52 full text articles were then assessed for eligibility. As 24 of them met exclusion criteria, only 28 articles were further analysed. These articles were published in 18 journals from 1959 to 2015, with 25 being original studies, and one randomised control trial. The total corpus included 18 prospective studies, 7 retrospective studies and 3 case reports.

KS is a significant complex, multidisciplinary clinical problem. The heterogeneous nature of the majority of published studies prevents unequivocal conclusions being drawn. Despite a great progress in knowledge about the respiratory system functioning and pathology in KS, the treatment seems to be not yet quite satisfactory. Therefore, there is a strong need for large prospective studies and unified clinical guidelines on the management of this group of patients.
\end{abstract}

Key words: kyphoscoliosis, respiratory failure, non-invasive ventilation, NIV, treatment

Adv. Respir. Med. 2017; 85: 352-357

\section{Introduction}

Kyphoscoliosis (KS) is an excessive curvature of the spine in the coronal and sagittal plane caused by vertebral anomalies [1]. It affects about $1 \%$ of the population but only $10 \%$ of the cases present significant clinical symptoms. Most KS cases are mild and have no impact on everyday life of the patient [2]. The deformity is probably known from antiquity - the first description of kyphoscoliosis probably dates from about $400 \mathrm{BC}$, as given by Hippocrates [2]. Moreover, KS is believed to be present in ancient Egyptian mummies, basing on an analysis of King Tutankhamun's family [3].

The aetiology of KS remains uncertain. It may eventuate due to traumatic injury in childhood, tuberculosis of the spine, or post-rachitic or neu- romuscular kyphoscoliosis due to poliomyelitis in infancy; however, the most common form is idiopathic kyphoscoliosis [4, 5].

Patients with KS have a characteristic body appearance characterised by a short stature due to reduction of the rib cage and trunk, and the presence of a hump created by the deformed thoracic cavity. However, the head, neck and limbs are normal in their length. The severity of KS is judged by the Cobb angle, which can be estimated by the angle formed by the intersection of two lines: a perpendicular to a line drawn across the superior endplate of the upper-end vertebra, and the inferior endplate of the lower-end vertebra of the deformity [5]. The most extreme deformities have been observed due to tuberculosis KS and polio KS [2]. The patients demonstrate a greater

Address for correspondence: Mateusz Pajdziński, Department of Pneumology and Allergy, $1^{\text {st }}$ Chair of Internal Medicine, Medical University of Lodz, Kopcińskiego 22,

90-153 Łódź, e-mail: mateusz.pajdzinski@gmail.com

DOI: 10.5603/ARM.2017.0060

Received: 20.06 .2017

Copyright (C) 2017 PTChP

ISSN 2451-4934 


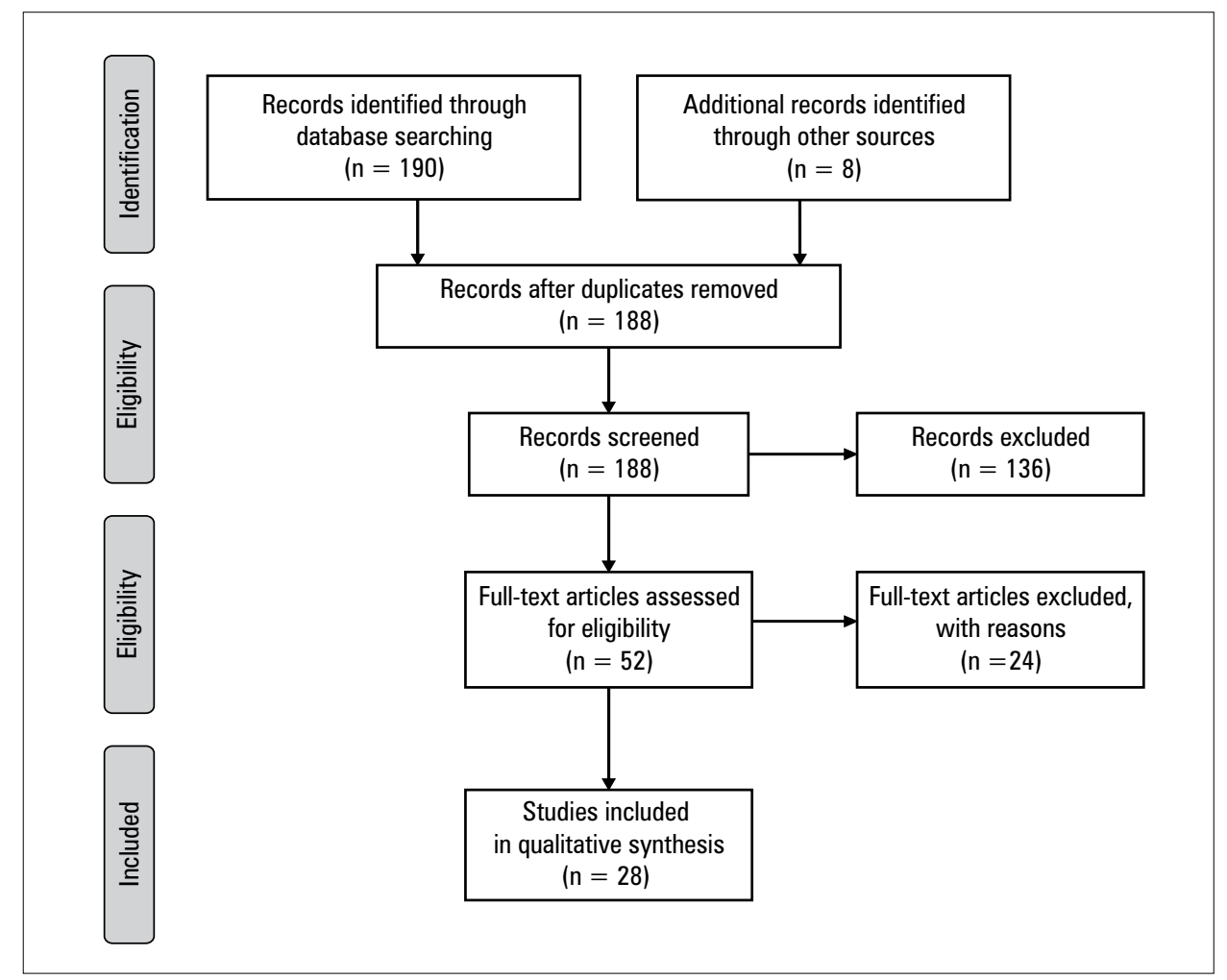

Figure 1. Flow chart of papers selection for inclusion to the study [8]

risk of muscle fatigue associated with ageing, and their chest wall compliance decreases [1]. These changes lead to impairment of the respiratory system and cause hypoventilation, hypercapnia and pulmonary hypertension, finally resulting in chronic respiratory failure (CRF) [6, 7]. These foregoing conditions affect the deterioration of health-related quality of life (HRQL). HRQL is an important clinical indicator, which should be taken into account while choosing therapy in patients with chronic problems [6]. There are no strict indications for surgical intervention; however, it should be performed in an early phase of the illness to prevent the exacerbation of deformation and development of severe lung disorders [1].

Kyphoscoliosis is a significant clinical problem and there are no precise guidelines for management, especially concerning respiratory pathology. Therefore, the aim of our study was to perform a systematic review summarising the present state of knowledge in the field.

\section{Material and methods}

The search included records dated before November 10, 2016 in the Pubmed database. The search term employed was "kyphoscoliosis and respiratory failure"; yielding 190 results being obtained. From these articles, 180 were chosen that were connected with the subject of study: A total of 136 articles related to orthopaedics, paediatrics, neurology, surgery and genetics were excluded. Of the 52 remaining studies, 24 were based on mixed groups, making difficult to draw unequivocal conclusions about patients with kyphoscoliosis (Fig. 1) [8], and the remaining 28 were passed to the next stage of analysis. In addition, another eight articles from the references were added. All reviewed articles were published in the period from 1959 to 2015 and came from 18 various journals. Twenty-five of them were original studies, with one randomised control trial. The corpus included 18 prospective studies, 7 retrospective studies and 3 case reports (Fig. 1).

\section{Lung function and mechanics of the respiratory system}

$\mathrm{KS}$, as a deformity of the thoracic wall, may influence mechanics of the respiratory system and lung function. Bergofsky et al. [2] have reported three main anatomical changes in the lung: chronic pulmonary emphysema with bronchial obstruction, changes in the small vessels of the lung and tangled and compressed vessels. Due 
to these changes, patients report the presence of coughing, sputum and wheezing, which can indicate asthma or bronchitis [2].

Increased residual volume (RV) to total lung capacity (TLC) ratio was also reported in cases of KS; this can be caused by a significant reduction in TLC and slight reduction in RV. Therefore, the nature of the above mentioned pathology is different from that observed in emphysema or chronic obstructive pulmonary disease, in which $\mathrm{RV}$ is found to be higher than normal [2].

Conti et al. [5] have reported on a reduction in the respiratory system compliance (CRS) by about $30 \%$, and a substantial increase in the ohmic and viscoelastic resistance component of the respiratory system in patients with KS compared with a control group. An increase in total resistance in the respiratory system results mainly from increased tissue resistance and marginally from a reduction in the airway size. The airway resistance did not respond to administration of doxophylline at a dose of $6 \mathrm{mg} / \mathrm{kg}$, which suggests that it has been induced by irreversible factors, such as airway deformity and retention of fluids. The authors have noted no correlation between compliance and resistance values and the amount of inspired oxygen [5].

There is also evidence that patients with KS have weakened functioning of the respiratory muscles, which may be confirmed by the measurement of transdiaphragmatic pressure during normal breathing and during maximal inspiration [7]. A correlation has been found between this pressure and abnormalities in gas exchange $[5,9]$. Reduction in TLC and vital capacity (VC) can be observed during the progression of the disease; these are caused by a gradual decline in respiratory system compliance, modification of the position of the chest wall and mechanical impairment of the respiratory muscles [10].

It is worth emphasising that McMaster et al. have found no statistically significant correlation between the size of the Cobb angle and the impairment of the lung function [1]. Similarly, Conti et al. have reported no connection between the Cobb angle and vital capacity values and mechanics variables [5]. However, when scoliosis and kyphosis were considered separately, a correlation was found between the Cobb angle of kyphosis and the failure of the respiration [1]. A major impairment of lung function is often observed when the kyphotic parts of the deformity predominate, probably caused by forward angulation of the spine and compression of the ribs on both sides, thus impairing the movements of the diaphragm [1]. Tests based on forced vital capacity (FVC) and forced expiratory volume in one second $\left(\mathrm{FEV}_{1}\right)$ have found the height of the apex of the kyphotic part of the kyphoscoliosis to be negatively associated with the results of the respiratory function tests, i.e. a higher apex is related to a poorer result [1].

\section{Exercise training and respiratory rehabilitation}

Many patients with kyphoscoliosis exhibit exercise limitation even though they demonstrate a normal breathing response to physical effort. Studies have shown that intolerance of exercise may arise from physical impairment [11]. The six-minute walking test (6MWT) is a simple way to assess endurance and exercise limits. The most important factor that affects the outcome of the 6MWT is dyspnoea at the beginning of the test. The Borg scale for dyspnoea is significantly negatively correlated with the outcomes. In spirometry, only $\mathrm{FEV}_{1}$ has been found to correlate with six-minute walking distance (6MWD), and this relationship is weak negative. Body mass index (BMI) and age have been negatively correlated with 6MWD, but no correlation has been found with the level of arterial blood gases. Karakurt et al. [12] have reported that patients with kyphoscoliosis walked a much shorter distance than they ought to (median $270 \mathrm{~m}-43.7 \%$ of predicted distance) [12]. Oxygen supplementation at the beginning and during the 6MWT did not elongate 6MWD, but long-term therapy with non-invasive ventilation (NIV) was found to have a significant positive effect [4]. Also, no correlation was found between 6MWD and initial desaturation, or between 6MWD and the size of Cobb angle. There is evidence that supplementation of oxygen can only improve the score of Visual Analogue Scale (VAS) for breathlessness and recovery time after exertion, but not the results of 6MWT [10].

A randomised control trial by Cejudo et al. [13] examined the influence of exercise on endurance and strength. A group of $34 \mathrm{KS}$ patients receiving night time home mechanical ventilation (NHMV) were divided randomly into two equally-sized groups: an exercise group and a control group. Pulmonary function, exercise capacity, peripheral muscle strength, dyspnoea and HRQL were measured. The exercise group subjects trained three non-consecutive days per week for 12 weeks. The training session was supervised and consisted of a 10-minute warm-up, 30 minutes of leg exercises on an ergometer cycle and 20 minutes of upper and lower body strength exercises. The authors 
reported significant decrease in $\mathrm{PaCO}_{2}$, while maximum inspiratory and expiratory pressures, as well as endurance time and shuttle walk distance increased significantly. The exercise group had also significantly greater improvements in peripheral muscle strength, dyspnoea, and quality of life [13].

Cejudo et al. [13] have recommended this kind of exercise programme for the treatment of chronic respiratory failure due to kyphoscoliosis in patients receiving NHMV.

In contrast, Fuschillo et al. [14] have found that the combination of pulmonary rehabilitation with non-invasive ventilation and long-term oxygen therapy improved peripheral muscle strength, HRQL and endurance only for a short period of time after the rehabilitation programme. They have reported that rehabilitation after a one-year follow-up did not prevent the test results decreasing. There is clearly a need to determine how to best optimise training to increase and maintain these benefits for a longer time.

It has also been revealed that the use of NIV during exercise decreases effort and fatigue. Patients who did not use ventilation experienced more significant acidosis, hypoxia, hypercapnia and dyspnoea [15].

\section{Cardiovascular system}

The deformation may significantly influence cardiovascular system dysfunction as well. Bergofsky et al. [2] have observed a positive correlation between age, severity of the deformity and the occurrence of cardiological diseases, especially in patients aged over 40 and with the Cobb angle greater than 100 degrees.

Cardiovascular problems are caused by changes in the respiratory system. The presence of an imbalance between perfusion and diffusion of the lung due to deformity of the rib cage and decrease in the total lung capacity leads to hypoxaemia. It causes hypertrophy and hyperplasia of the medial layer of the pre-capillary vessels, causing pulmonary hypertension, with the resulting hypertrophy of the right side of the heart being observed in most autopsies of KS patients. The second most common cause of cardiac failure comprises the kinking and compression of the great vessels as a result of the rib cage deformity [2].

\section{Pregnancy and delivery}

Although this review is focused on the respiratory system, some issues about pregnancy and delivery should be mentioned as being clinically significant for the topic. Kyphoscoliosis is an uncommon deformation in pregnancy; it is estimated that only $0.02 \%$ to $0.7 \%$ of pregnant women suffer from this condition [16, 17]. However, patients experience several complications due to the abnormal curvature of the spine, these being classed as either medical or obstetric complications. The former consist of pulmonary restriction, acute respiratory failure (ARF) and CRF, cor pulmonale and heart failure. As the greatest risk of pulmonary function deterioration usually occurs in the third trimester, monitoring of arterial blood gases and overnight oxymetry should be performed to identify when ventilation support should be commenced [18]. Berman et al. [19] have indicated that pregnancy exacerbates scoliosis due to mechanical stress and pregnancy hormones; hence, Wong et al. [16] have suggested reassessment of the spinal curvature after delivery. Obstetric complications include gestation hypertension, preeclampsia, preterm delivery and a higher risk of C-section [15, 16]. Chopra et al. [17] have noticed that all delivered babies had good Apgar scores and any of the babies had congenital malformations. Few complications have been recorded during delivery, such as breech presentation [17] and deep transverse arrest [20]. In such cases, it is recommended that C-section is performed under general anaesthesia as the changes in spinal curvature and epidural space complicate correct needle placement [21].

\section{Treatment of acute respiratory failure}

Infections of the airways can lead to dangerous destabilisation of the respiratory tract homeostasis, especially in patients with KS. It increases the risk of ARF and the necessity of hospitalisation. Banfi et al. [22] have showed that KS patients with infection-related ARF can be treated successfully and safely at home. In their study, seven patients with ARF were treated with home mechanical ventilation (HMV) increased to 20 hours/day and proper antibiotics. The patients progressively decreased the daily time of mechanical ventilation according to their ability to breathe comfortably. After four weeks they achieved the same weekly duration of mechanical ventilatory assistance as observed before the ARF episode; however, it is important to note that HMV can only act as a support treatment for an antibiotics regimen [22].

The most common reason for admission to intensive care unit (ICU) due to ARF in patient 
with KS was the occurrence of cor pulmonale and sepsis. Sepsis was typically associated with pneumonia, but was on rare occasions caused by other infections like lower respiratory tract infections and urinary tract infections: these conditions being indications for treatment in the ICU [4] Several studies have compared the use of NIV and invasive mechanical ventilation (IMV) in KS patients with ARF. The choice of ventilation support method depends on the condition of the patient during admission to the ICU. Lower $\mathrm{pH}$ values at admission, occurrence of sepsis, compulsion of using IMV and failure of NIV usage are indicators of worse prognosis for ARF patients.

The first to describe the influence of ventilation on the condition of patients during ARF was the study performed in 1995 by Finlay et al. [23], who identified improved blood oxygen concentration $\left(\mathrm{PaO}_{2}\right)$ and reduced blood carbon dioxide concentration $\left(\mathrm{PaCO}_{2}\right)$ after nasal intermittent positive pressure ventilation. NIV also ameliorated $\mathrm{FEV}_{1}$ and FVC, as well as $\mathrm{FEV}_{1}$ after discharge from hospital. Indicators for NIV failure include lower $\mathrm{pH}$ and lower $\mathrm{PaO}_{2} / \mathrm{FiO}$ ratio after one hour of NIV usage [4]. Patients with successful treatment with NIV and those who are initially intubated are much less likely to develop sepsis or to die than patients with NIV failure. Adigüzel et al. [4] strongly discourage the use of NIV in the case of sepsis and septic shock.

\section{Treatment of chronic respiratory failure and sleep disorders}

NIV is now regarded as an option of treating CRF in patients with KS [4]. Gustafson et al. [24] also have reported that the survival rate of patients using home mechanical ventilation was three times higher than that of patients using long-term oxygen therapy alone (LTOT). Patients with KS are more exposed to hypoventilation during sleep. It can lead to hypoxaemia and hypercapnia, which can cause pulmonary hypertension and can weaken muscle strength. Sawicka et al. [25] have noted that the length of the each phase of sleep is correct, but longer periods of sleep are associated with greater desaturation, especially during rapid eye movement (REM) sleep. Desaturations were mainly caused by earlier episodes of hypopnea, and to a lesser degree, by changes in the chest movements that did not meet the criteria of hypopnea resulting from dysfunction of the diaphragm [25]. REM sleep is characterised by the greatest end tidal carbon dioxide tension, which is reflected in a greater frequency of hypopnea. Although no correlations have been found between desaturation and the Cobb angle size, a relationship has been seen between desaturation, patient age and basal vital capacity. It is not possible to relate this knowledge to all patients with KS because most have comorbidities, which can affect breathing during sleep. The most common conditions that can deteriorate breathing problems during sleep are obesity and systemic hypertension, as well as associated obstructive sleep apnoea. The ideal weight for height in patients with $\mathrm{KS}$ is difficult to determine. On the basis of true height, ideal weight would tend to be underestimated [25].

The choice of treatment depends on the comorbidities and underlying condition. If the patient has obstructive disorders, continuous positive airways pressure (CPAP) is recommended; however, this may not be sufficient in other kinds of respiratory pathology. Then, NIV is recommended. After three months of NIV in KS patients, Ellis et al. [26] have found that the length and quality of REM sleep improved, while oxygenation increased and transcutaneous carbon dioxide tension decreased. After NIV, daytime arterial blood gas results improvement and hospitalisation rate decrease were observed as well [26, 27]. However, NIV does not improve spirometry parameters [27]. After termination of NIV for a few nights, patients again complained about sleepiness, breathlessness during sleep due to the results of the tests, which were inappropriate. Buyse et al. [28] have identified a higher life expectancy in patients with CRF treated with combined LTOT with nocturnal nasal intermittent positive pressure ventilation (nNIPPV) than those treated with LTOT alone: ABG results and vital capacity were both improved [28, 29]. Other studies have shown that nNIPPV improves $\mathrm{PaO}_{2}$ and $\mathrm{PaO}_{2} / \mathrm{FiO}_{2}$ ratio, as well as FVC and respiratory muscle strength [7]. The use of this type of ventilation support decreased the frequency of hospitalisation: no admissions to the pulmonology ward were required due to respiratory failure during the course of therapy. Effects were observed after six months of therapy [7].

\section{Health-related quality of life}

Patients with KS complain about chronic fatigue, dyspnoea, aggravating functional status and frequent readmission to hospital, all of which decrease the quality of life. Cejudo et al. [6] have found that tiredness had the most significant impact on HRQL, followed by peripheral muscle 
strength, dyspnoea and exercise capacity. They recommend the use of home mechanical ventilation and a suitable training programme.

\section{Summary}

$\mathrm{KS}$ is a significant complex, multidisciplinary clinical problem. The results of the majority of published studies are heterogeneous, which currently prevents unequivocal conclusions from being drawn. Coming back to the question asked in the title: what can we do for respiration besides NIV? The answer is: little. Although the deformity is probably known from antiquity, and despite a great progress in knowledge about the respiratory system functioning and pathology in KS, the treatment seems to be not yet quite satisfactory. Therefore, there is a strong need for large control trials and unified clinical guidelines on the management of this group of patients.

\section{Conflict of interest}

The authors declares no conflict of interest.

\section{References:}

1. McMaster MJ, Glasby MA, Singh H, et al. Lung function in congenital kyphosis and kyphoscoliosis. J Spinal Disord Tech. 2007; 20(3): 203-208, doi: 10.1097/01.bsd.0000211270.51368.43, indexed in Pubmed: 17473639.

2. Bergofsky EH, Turino GM, Fishman AP. Cardiorespiratory failure in kyphoscoliosis. Medicine (Baltimore). 1959; 38: 263-317, indexed in Pubmed: 13799416.

3. Hawass Z, Gad YZ, Ismail S, et al. Ancestry and pathology in King Tutankhamun's family. JAMA. 2010; 303(7): 638-647, doi: 10.1001/jama.2010.121, indexed in Pubmed: 20159872.

4. Adıüzel N, Karakurt Z, Güngör G, et al. Management of kyphoscoliosis patients with respiratory failure in the intensive care unit and during long term follow up. Multidiscip Respir Med. 2012; 7(1): 30, doi: 10.1186/2049-6958-7-30, indexed in Pubmed: 22999093.

5. Conti G, Rocco M, Antonelli M, et al. Respiratory system mechanics in the early phase of acute respiratory failure due to severe kyphoscoliosis. Intensive Care Med. 1997; 23(5): 539-544, indexed in Pubmed: 9201526.

6. Cejudo P, López-Márquez I, Lopez-Campos JL, et al. Factors associated with quality of life in patients with chronic respiratory failure due to kyphoscoliosis. Disabil Rehabil. 2009; 31(11): 928-934, doi: 10.1080/09638280802358316, indexed in Pubmed: 19116808.

7. Gonzalez C, Ferris G, Diaz J, et al. Kyphoscoliotic ventilatory insufficiency: effects of long-term intermittent positive-pressure ventilation. Chest. 2003; 124(3): 857-862, indexed in Pubmed: 12970009.

8. Moher D, Liberati A, Tetzlaff J, et al. PRISMA Group, PRISMA Group, PRISMA Group, PRISMA Group, PRISMA Group, PRISMA Group, PRISMA Group. Preferred reporting items for systematic reviews and meta-analyses: the PRISMA Statement. Open Med. 2009; 3(3): e123-e130, indexed in Pubmed: 21603045.

9. Lisboa C, Moreno R, Fava M, et al. Inspiratory muscle function in patients with severe kyphoscoliosis. Am Rev Respir Dis. 1985; 132(1): 48-52, doi: 10.1164/arrd.1985.132.1.48, indexed in Pubmed: 4014872.

10. Jones DJ, Paul EA, Bell JH, et al. Ambulatory oxygen therapy in stable kyphoscoliosis. Eur Respir J. 1995; 8(5): 819-823, indexed in Pubmed: 7656956.
11. Kesten S, Garfinkel SK, Wright T, et al. Impaired exercise capacity in adults with moderate scoliosis. Chest. 1991; 99(3): 663-666, indexed in Pubmed: 1995222.

12. Karakurt Z, Güven AO, Moçin OY, et al. Six minute walking distance in kyphoscoliosis patients with chronic respiratory failure. Multidiscip Respir Med. 2010; 5(4): 244-249, doi: 10.1186/2049-6958-5-4-244, indexed in Pubmed: 22958538.

13. Cejudo P, López-Márquez I, López-Campos JL, et al. Exercise training in patients with chronic respiratory failure due to kyphoscoliosis: a randomized controlled trial. Respir Care. 2014; 59(3): 375-382, doi: 10.4187/respcare.02484, indexed in Pubmed: 23983272.

14. Fuschillo S, De Felice A, Martucci M, et al. Pulmonary rehabilitation improves exercise capacity in subjects with kyphoscoliosis and severe respiratory impairment. Respir Care. 2015; 60(1): 96-101, doi: 10.4187/respcare.03095, indexed in Pubmed: 25228781.

15. Vila B, Servera E, Marín J, et al. Noninvasive ventilatory assistance during exercise for patients with kyphoscoliosis: a pilot study. Am J Phys Med Rehabil. 2007; 86(8): 672-677, doi: 10.1097/PHM.0b013e31806dd2c8, indexed in Pubmed: 17667198.

16. To W, Wong M. Kyphoscoliosis complicating pregnancy. International Journal of Gynecology \& Obstetrics. 1999; 55(2): 123-128, doi: 10.1016/s0020-7292(96)02745-2.

17. Chopra S, Adhikari K, Agarwal N, et al. Kyphoscoliosis complicating pregnancy: maternal and neonatal outcome. Arch Gynecol Obstet. 2011; 284(2): 295-297, doi: 10.1007/s00404010-1638-3, indexed in Pubmed: 20711597.

18. Reddy R, Evans E, Khoo O, et al. Pregnancy in kyphoscoliosis: benefit of non-invasive ventilatory support. J Obstet Gynaecol. 2005; 25(3): 267-268, doi: 10.1080/01443610500060867, indexed in Pubmed: 16147732.

19. Berman AT, Cohen DL, Schwentker EP. The effects of pregnancy on idiopathic scoliosis. A preliminary report on eight cases and a review of the literature. Spine (Phila Pa 1976). 1982; 7(1): 76-77, indexed in Pubmed: 7071665.

20. Jones DH. Kyphoscoliosis complicating pregnancy. Obstetrical \& Gynecological Survey. 1964; 19(4): 617-618, doi: 10.1097/00006254-196408000-00011.

21. Kuczkowski KM. Labor analgesia for the parturient with an uncommon disorder: a common dilemma in the delivery suite. Obstet Gynecol Surv. 2003; 58(12): 800-803, doi: 10.1097/01. OGX.0000097782.52780.3E, indexed in Pubmed: 14668661.

22. Banfi P, Redolfi S, Robert D. Home treatment of infection-related acute respiratory failure in kyphoscoliotic patients on long-term mechanical ventilation. Respir Care. 2007; 52(6): 713-719, indexed in Pubmed: 17521460.

23. Finlay G, Concannon D, McDonnell TJ. Treatment of respiratory failure due to kyphoscoliosis with nasal intermittent positive pressure ventilation (NIPPV). Ir J Med Sci. 1995; 164(1): 28-30, indexed in Pubmed: 7890530.

24. Gustafson T, Franklin KA, Midgren B, et al. Survival of patients with kyphoscoliosis receiving mechanical ventilation or oxygen at home. Chest. 2006; 130(6): 1828-1833, doi: 10.1378/ chest.130.6.1828, indexed in Pubmed: 17167004.

25. Sawicka EH, Branthwaite MA. Respiration during sleep in kyphoscoliosis. Thorax. 1987; 42(10): 801-808, indexed in Pubmed: 3424256.

26. Ellis ER, Grunstein RR, Chan S, et al. Noninvasive ventilatory support during sleep improves respiratory failure in $\mathrm{ky}$ phoscoliosis. Chest. 1988; 94(4): 811-815, indexed in Pubmed: 3139373.

27. Nauffal D, Doménech R, Martínez García MA, et al. Noninvasive positive pressure home ventilation in restrictive disorders: outcome and impact on health-related quality of life. Respir Med. 2002; 96(10): 777-783, indexed in Pubmed: 12412976.

28. Buyse B, Meersseman W, Demedts M. Treatment of chronic respiratory failure in kyphoscoliosis: oxygen or ventilation? Eur Respir J. 2003; 22(3): 525-528, indexed in Pubmed: 14516146.

29. Piesiak P, Brzecka A, Kosacka M, et al. Efficacy of noninvasive volume targeted ventilation in patients with chronic respiratory failure due to kyphoscoliosis. Adv Exp Med Biol. 2015; 838: 53-58, doi: 10.1007/5584_2014_68, indexed in Pubmed: 25315620 . 